International Journal of Pure and Applied Mathematics

Volume 88 No. 4 2013, 569-575

ISSN: 1311-8080 (printed version); ISSN: 1314-3395 (on-line version)

url: http://www.ijpam.eu

doi: http://dx.doi.org/10.12732/ijpam.v88i4.11

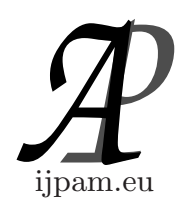

\title{
ON DETERMINANTS OF SOME TRIDIAGONAL MATRICES CONNECTED WITH FIBONACCI NUMBERS
}

\author{
Jiří Jína ${ }^{1}$, Pavel Trojovský ${ }^{2} \S$ \\ ${ }^{1,2}$ Department of Mathematics \\ Faculty of Science \\ University of Hradec Králové \\ Rokitanského 62, 50003 Hradec Králové, CZECH REPUBLIC
}

\begin{abstract}
We will overview some facts about the Fibonacci numbers, Hessenberg matrices and tridiagonal matrices. We will summarize the results on determinants of families of tridiagonal matrices which are equal to a Fibonacci number, but we prove most of this results by simpler and more direct way with the help of The On-line Encyclopedai of Integer Sequences (OEIS).
\end{abstract}

AMS Subject Classification: 11B39, 15A15

Key Words: matrix, tridiagonal matrix, Hessenbeg matrix, Toeplitz matrix, recurrence

\section{Introduction}

Fibonacci sequence (or sequence of the Fibonacci numbers) $\left\langle F_{n}\right\rangle_{n=0}^{\infty}$ is the sequence of positive integers satisfying the recurrence relation $F_{n+2}=F_{n+1}+F_{n}$ with the initial conditions $F_{0}=0$ and $F_{1}=1$. Lucas sequence is a sequence $\left\langle L_{n}\right\rangle_{n=0}^{\infty}$ of positive integers satisfying the same recurrence as the Fibonacci numbers but with the initial conditions $L_{0}=2$ and $L_{1}=1$. These sequences are usually extended for negative indices by identities $F_{-n}=(-1)^{n+1} F_{n}$ and $L_{-n}=(-1)^{n} L_{n}$.

Received: September 19, 2013

(C) 2013 Academic Publications, Ltd.

$\S$ Correspondence author url: www.acadpubl.eu 
For the Fibonacci and Lucas numbers was derived many identities (see [6] or $[10])$, e. g. the following helpful identity

$$
F_{k+n}=L_{n} F_{k}+(-1)^{n+1} F_{k-n} .
$$

The lower Hessenberg triangular matrix $\mathbb{M}(n)$ type $n \times n$, where $m_{j k}$ are any real numbers and $m_{j k}=0$ for $k>j+1$, thus

$$
\mathbb{M}(n)=\left(\begin{array}{cccccc}
m_{11} & m_{12} & 0 & 0 & \cdots & 0 \\
m_{21} & m_{22} & m_{23} & 0 & \cdots & 0 \\
m_{31} & m_{32} & m_{33} & m_{34} & \ddots & \vdots \\
\vdots & \vdots & \vdots & \ddots & \ddots & 0 \\
m_{n-1,1} & m_{n-1,2} & m_{n-1,3} & \cdots & m_{n-1, n-1} & m_{n-1, n} \\
m_{n 1} & m_{n 2} & m_{n 3} & \cdots & m_{n, n-1} & m_{n n}
\end{array}\right) .
$$

Cahill et al. in [3] proved the following recurrence for the determinant of lower Hessenberg matrix

$$
\begin{aligned}
\operatorname{det} \mathbb{M}(0)= & 1, \operatorname{det} \mathbb{M}(1)=m_{11}, \\
\operatorname{det} \mathbb{M}(2)= & m_{11} m_{22}-m_{12} m_{21}, \\
\operatorname{det} \mathbb{M}(n)= & m_{n n} \operatorname{det} \mathbb{M}(n-1) \\
& +\sum_{r=1}^{n-1}\left((-1)^{n-r} m_{n r} \operatorname{det} \mathbb{M}(r-1) \prod_{j=r}^{n-1} m_{j, j+1}\right)
\end{aligned}
$$

\section{The Tridiagonal Matrices}

In this section we recall the basic recurrence for the determinant of the tridiagonal matrix and then we show using of it for special cases of tridiagonal matrices.

Matrix $\mathbb{A}(n)$ is called a tridiagonal matrix, when it is in the following form 


$$
\mathbb{A}(n)=\left(\begin{array}{ccccccc}
a_{11} & a_{12} & 0 & 0 & \cdots & 0 & 0 \\
a_{21} & a_{22} & a_{23} & 0 & \cdots & 0 & 0 \\
0 & a_{32} & a_{33} & a_{34} & \ddots & \vdots & \vdots \\
\vdots & 0 & \ddots & \ddots & \ddots & 0 & 0 \\
0 & \vdots & \ddots & \ddots & \ddots & a_{n-2, n-1} & 0 \\
0 & 0 & \cdots & 0 & a_{n-1, n-1} & a_{n-1, n-1} & a_{n-1, n} \\
0 & 0 & 0 & \cdots & 0 & a_{n, n-1} & a_{n n}
\end{array}\right)
$$

Setting $m_{i j}=0$ for $j>i+1$ and $i>j+1$ in (2) we obtain for determinant of tridiagonal matrix the following recurrence

$$
\begin{aligned}
\operatorname{det} \mathbb{A}(0) & =1, \quad \operatorname{det} \mathbb{A}(1)=a_{11}, \\
\operatorname{det} \mathbb{A}(2) & =a_{11} a_{22}-a_{12} a_{21}, \\
\operatorname{det} \mathbb{A}(n) & =a_{n n} \operatorname{det} \mathbb{A}(n-1)-a_{n, n-1} a_{n-1, n} \operatorname{det} \mathbb{A}(n-2) .
\end{aligned}
$$

Now we turn our attention to the relation between determinant of special tridiagonal matrix with the Fibonacci numbers. Most of presented matrices in the text bellow is connected with Toeplitz matrix (or diagonal-constant matrix), see [4]. The first example was probably done by Strang in [9], where he showed, that the determinant of $n \times n$ matrix

$$
\mathbb{B}(n)=\left(\begin{array}{ccccccc}
1 & -1 & 0 & \cdots & 0 & 0 & 0 \\
1 & 1 & -1 & 0 & \cdots & 0 & 0 \\
0 & 1 & 1 & -1 & 0 & \cdots & 0 \\
\vdots & 0 & 1 & 1 & \ddots & \ddots & \vdots \\
0 & \vdots & \ddots & \ddots & \ddots & -1 & 0 \\
0 & 0 & \cdots & 0 & 1 & 1 & -1 \\
0 & 0 & 0 & \cdots & 0 & 1 & 1
\end{array}\right)
$$

is equal to $F_{n}$ for $n \geq 1$. This result clearly follows from (3) setting $a_{n n}=$ $a_{n, n-1}=1$ and $a_{n-1, n}=-1$.

Similarly, setting $a_{n n}=1$ and $a_{n, n-1}=a_{n-1, n}=i=\sqrt{-1}$ in (3) we obtain 
that for the determinant of matrix

$$
\mathbb{C}(n)=\left(\begin{array}{ccccccc}
1 & i & 0 & \cdots & 0 & 0 & 0 \\
i & 1 & i & 0 & \cdots & 0 & 0 \\
0 & i & 1 & i & 0 & \cdots & 0 \\
\vdots & 0 & i & 1 & \ddots & \ddots & \vdots \\
0 & \vdots & \ddots & \ddots & \ddots & i & 0 \\
0 & 0 & \cdots & 0 & i & 1 & i \\
0 & 0 & 0 & \cdots & 0 & i & 1
\end{array}\right)
$$

the recurrence

$$
\begin{aligned}
\operatorname{det} \mathbb{C}(1) & =1, \operatorname{det} \mathbb{C}(2)=2, \\
\operatorname{det} \mathbb{C}(n) & =\operatorname{det} \mathbb{C}(n-1)+\operatorname{det} \mathbb{C}(n-2)
\end{aligned}
$$

holds. So we have $\operatorname{det} \mathbb{C}(n)=F_{n+1}, n \geq 1$.

Another example was described by Cahill et al. in [1], their considered the following matrix type $n \times n$

$$
\mathbb{D}(n)=\left(\begin{array}{ccccccc}
3 & -1 & 0 & \cdots & 0 & 0 & 0 \\
-1 & 3 & -1 & 0 & \cdots & 0 & 0 \\
0 & -1 & 3 & -1 & 0 & \cdots & 0 \\
\vdots & 0 & -1 & 3 & \ddots & \ddots & \vdots \\
0 & \vdots & \ddots & \ddots & \ddots & -1 & 0 \\
0 & 0 & \cdots & 0 & -1 & 3 & -1 \\
0 & 0 & 0 & \cdots & 0 & -1 & 3
\end{array}\right) .
$$

Setting $a_{n n}=3$ and $a_{n, n-1}=a_{n-1, n}=-1$ in (3) can be obtained for the determinant of matrix $\mathbb{D}(n)$ the following recurrence

$$
\begin{aligned}
\operatorname{det} \mathbb{D}(1) & =3, \quad \operatorname{det} \mathbb{D}(2)=8, \\
\operatorname{det} \mathbb{D}(n) & =3 \operatorname{det} \mathbb{D}(n-1)-\operatorname{det} \mathbb{D}(n-2) .
\end{aligned}
$$

The sequence defined by the previous recurrence is named A001906 in [8], therefore $\operatorname{det} \mathbb{D}(n)=F_{2(n+1)}$ for all $n \geq 1$. Cahill et al. in [1] also showed, that the determinant does not change if we replace -1 by 1 on superdiagonal and subdiagonal in the matrix $\mathbb{D}(n)$. 
Kilic and Tasci [5] showed that the tridiagonal matrix

$$
\mathbb{E}(n)=\left(\begin{array}{ccccccc}
-1 & 1 & 0 & \cdots & 0 & 0 & 0 \\
-1 & -1 & 1 & 0 & \cdots & 0 & 0 \\
0 & -1 & -1 & 1 & 0 & \cdots & 0 \\
\vdots & 0 & -1 & -1 & \ddots & \ddots & \vdots \\
0 & \vdots & \ddots & \ddots & \ddots & 1 & 0 \\
0 & 0 & \cdots & 0 & -1 & -1 & 1 \\
0 & 0 & 0 & \cdots & 0 & -1 & -1
\end{array}\right)
$$

is connected with the Fibonacci numbers with negative indexes, as setting $a_{n n}=$ $-1, a_{n, n-1}=-1$ and $a_{n-1, n}=1$ in (3) can be obtained for the determinant of matrix $\mathbb{E}(n)$ the following recurrence

$$
\begin{aligned}
\operatorname{det} \mathbb{E}(1) & =-1, \quad \operatorname{det} \mathbb{E}(2)=2, \\
\operatorname{det} \mathbb{E}(n) & =-\operatorname{det} \mathbb{E}(n-1)+\operatorname{det} \mathbb{E}(n-2),
\end{aligned}
$$

but we can found that the sequence defined by this recurrence is named $A 039834$ in [8], hence

$$
\operatorname{det} \mathbb{E}(n)=F_{-(n+1)}=(-1)^{n} F_{n+1} .
$$

A major shift in this topics was done by Cahill et al. [3] as they proved the theorem on a tridiagonal matrix with parameters $\alpha, \beta$, whose determinant is connected with the Fibonacci sequence. Let $\mathbb{F}_{\alpha, \beta}(n)$ be a family of symmetric tridiagonal matrices, where $\alpha, \beta$ are any positive integers, with entries

$$
f_{j k}=\left\{\begin{array}{cc}
F_{\alpha+\beta}, & j=1, k=1 ; \\
\left\lceil\frac{F_{2 \alpha+\beta}}{\left.F_{\alpha+\beta}\right\rceil,}\right. & j=2, k=2 ; \\
L_{\alpha}, & k=j, 3 \leq j \leq k ; \\
\sqrt{\left\lceil\frac{F_{2 \alpha+\beta}}{F_{\alpha+\beta}}\right\rceil F_{\alpha+\beta}-F_{2 \alpha+\beta}}, & j=1, k=2 \text { or } j=2, k=1 ; \\
\sqrt{(-1)^{\alpha}}, & k=j \pm 1,2 \leq j<k ; \\
0, & \text { other cases, }
\end{array}\right.
$$

that is

$$
\mathbb{F}_{\alpha, \beta}(n)=\left(\begin{array}{ccccc}
F_{\alpha+\beta} & f_{12} & 0 & \cdots & 0 \\
f_{21} & \left\lceil\frac{F_{2 \alpha+\beta}}{F_{\alpha+\beta}}\right] & \sqrt{(-1)^{\alpha}} & \ddots & \vdots \\
0 & \sqrt{(-1)^{\alpha}} & L_{\alpha} & \ddots & 0 \\
\vdots & \ddots & \ddots & \ddots & \sqrt{(-1)^{\alpha}} \\
0 & \cdots & 0 & \sqrt{(-1)^{\alpha}} & L_{\alpha}
\end{array}\right) .
$$


Cahill et al. in [3] proved using recurrence (3) and identity (1) that

$$
\operatorname{det} \mathbb{F}_{\alpha, \beta}(n)=F_{\alpha n+\beta}
$$

For example, setting $\alpha=4$ and $\beta=-2$ in (5) can be obtained that the determinant of the matrix

$$
\mathbb{F}_{4,-2}(n)=\left(\begin{array}{ccccccc}
1 & 1 & 0 & \cdots & 0 & \cdots & 0 \\
1 & 8 & 1 & 0 & \cdots & \ddots & \vdots \\
0 & 1 & 7 & 1 & 0 & \cdots & 0 \\
0 & 0 & \ddots & \ddots & \ddots & 0 & \vdots \\
0 & \ddots & 0 & 1 & 7 & 1 & 0 \\
\vdots & \ddots & \ddots & 0 & 1 & 7 & 1 \\
0 & \cdots & 0 & 0 & 0 & 1 & 7
\end{array}\right)
$$

is equal to $F_{4 n-2}$.

Nalli and Civciv [7] generalized results in [3] and showed for example, that the determinant of matrix

$$
\left(\begin{array}{ccccccc}
-1 & 0 & 0 & \cdots & 0 & \cdots & 0 \\
0 & -2 & i & 0 & \cdots & \ddots & \vdots \\
0 & i & -1 & i & 0 & \cdots & 0 \\
0 & 0 & \ddots & \ddots & \ddots & 0 & \vdots \\
0 & \ddots & 0 & i & -1 & i & 0 \\
\vdots & \ddots & \ddots & 0 & i & -1 & i \\
0 & \cdots & 0 & 0 & 0 & i & -1
\end{array}\right)
$$

is equal to $(-1)^{k} F_{k+1}$. This result easily follows from (3) as recurrence (4) again holds for the determinant of the previous matrix.

\section{Acknowledgments}

The authors thank to Specific research 2119 UHKCZ for financial support. 


\section{References}

[1] N. D. Cahil, J. R. D'Errico, D. A.Narayan, and J. Y. Narayan, Fibonacci determinants, College Math. J., 33, No. 3 (2002), 221-225

[2] N. D. Cahil, J. R. D'Errico, J. R., and J. P. Spence, Complex Factorizations of the Fibonacci and Lucas Numbers, Fib. Quart., 41, No. 1 (2003), 13-19.

[3] N. D. Cahill, D. A. Narayan, Fibonacci and Lucas numbers as tridiagonal matrix determinants. Fib. Quart., 42, No. 3 (2004), 216-221.

[4] R. M. Gray, Toeplitz and Circulant Matrices, A Review, now Publishers Inc., 2006.

[5] E. Kilic, D. Tasci, Negatively subscripted Fibonacci and Lucas numbers and their complex factorizations, Ars Combin., 96, (2010), 275-288.

[6] T. Koshy, Fibonacci and Lucas numbers with applications, John Wiley \& Sons, 2011.

[7] A. Nalli, H. Civciv, A generalization of tridiagonal matrix determinants, Fibonacci and Lucas numbers, Chaos, Solitons 85 Fractals, 40, No. 1 (2009), 355-361

[8] The OEIS Foundation Inc., The On-Line Encyclopedia of Integer Sequences, http://oeis.org (2013).

[9] G. Strang, Linear algebra and its applications, Brooks/Cole, 3rd edition (1988).

[10] S. Vajda, Fibonacci and Lucas Numbers, and the Golden Section, New York: Wiley (1989). 
